\title{
The Relationship between Adult Height and Blood Pressure
}

\author{
John Michael Cochran ${ }^{a, b}$ Vincent R. Siebert ${ }^{a, c}$ Jeffrey Bates ${ }^{a}$ Djenita Butulija ${ }^{a}$ \\ Anna Kolpakchi ${ }^{a}$ Himabindu Kadiyala ${ }^{a}$ Addison Taylor $^{a}$ Hani Jneid ${ }^{d}$ \\ aDepartment of Internal Medicine, Baylor College of Medicine and the Michael E DeBakey VA Medical Center, \\ Houston, TX, USA; ' Department of Cardiology, Baylor University Medical Center, Dallas, TX, USA; 'Department of \\ Cardiology, Loyola University Medical Center, Chicago, IL, USA; dSection of Cardiology, Baylor College of Medicine \\ and the Michael E DeBakey VA Medical Center, Houston, TX, USA
}

\section{Keywords}

Hypertension · Height · Blood pressure $\cdot$ Stature $\cdot$ Coronary artery disease

\begin{abstract}
Background: Identification and modification of cardiovascular risk factors is paramount to reducing cardiovascular disease morbidity and mortality. Hypertension is a major risk factor for cardiovascular disease, but its association with height remains largely underrecognized. Objectives: The objective of this manuscript is to review the evidence examining the association between blood pressure and human stature and to summarize the plausible pathophysiological mechanisms behind such an association. Methods: A systematic review of adult human height and its association with hypertension and coronary artery disease was undertaken. The literature evidence is summarized and tabulated, and an overview of the pathophysiological basis for this association is presented. Results: Shorter arterial lengths found in shorter individuals may predispose to hypertension in a complex hemodynamic interplay, which is explained predominantly by summated arterial wave reflections and an elevated augmentation index. Our systemic review sug-
\end{abstract}

gests that an inverse relationship between adult height and blood pressure exists. However, differences in the studied populations and heterogeneity in the methods applied across the various studies limit the generalizability of these findings and their clinical application. Conclusion: Physiological studies and epidemiological data suggest a potential inverse association between adult height and blood pressure. Further research is required to define the relationship more clearly between adult height and blood pressure and to assess whether antihypertensive therapeutic approaches and goals should be modified according to patients' heights.

(c) 2021 S. Karger AG, Basel

\section{Introduction}

Cardiovascular disease imparts an enormous global impact [1] with $>75$ million affected in the USA alone [2]. Risk factors for cardiovascular disease are identified and modified when feasible, and hypertension figures prominently among them. The latest research has stressed the importance of controlling hypertension, and the SPRINT trial [3] results shifted national and international guidelines towards stricter standards for arterial blood pressure

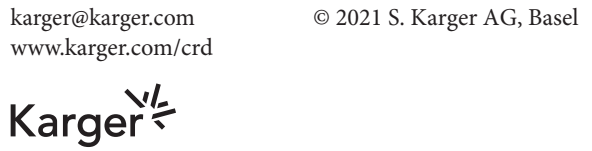


control. Obesity, smoking, lack of physical exercise, and dietary elements are all well-known modifiable risk factors [4]. Certain nonmodifiable risk factors, such as family history, age, and biological sex, are also well established. Height, while factored into the BMI formula, is a less recognized modifier for hypertension. The guideline statements from major cardiovascular disease associations, including the American College of Cardiology and the American Heart Association [5, 6], as well as the European Society of Cardiology and the European Society of Hypertension [7], do not include any reference to height as a modifier for patients' arterial blood pressure, except as it relates to their BMI or echocardiographic measurements of cardiac structures. The aims of the current manuscript are to review the evidence examining the association between blood pressure and human stature and to summarize the plausible pathophysiological mechanisms behind such an association.

\section{Pathophysiological Basis}

The pathophysiology of hypertension is multifaceted and complex, involving interplay between genetic predisposition, cardiovascular and arterial hemodynamics, structural/architectural changes of blood vessels, and neurohormonal cascades. The mechanism of hypertension often varies with the age of the patient. In children and younger adults, increased cardiac output is the primary contributor. In the middle aged, systemic vascular resistance plays a larger role, and in the elderly, stiffening of the major vessels (decreased compliance) factors heavily [8]. While the overarching mechanism of hypertension can be generalized by age range, there are many factors that may contribute to an individual patient's disease, including rare genetic disorders, environmental and lifestyle factors (dietary sodium, smoking, cardiovascular exercise, and alcohol), and neurohormonal (including renin-angiotensin axis) abnormalities [6].

Physiological evidence exists to explain lower blood pressure in taller people. Arterial wave reflection is important in the maintenance of systolic and diastolic blood pressure. LV ejection propagates a pressure wave along the arterial vasculature. When this pressure wave encounters arterial bifurcations or other anatomic variants that impede laminar flow, pressure wave reflections are generated [8]. Arterial compliance then determines the net effect - in younger patients with more elastic arteries and quicker recoil, the summation of wave reflections tends to augment the diastolic blood pressure, but in the elderly patient with stiffer arteries, the sum of wave reflections returns nearer to systole and contributes to a higher systolic and pulse pressure [9]. But, arterial compliance is only 1 factor in explaining the significance of arterial wave reflections and blood pressure; arterial length is also important. To demonstrate this, McEniery et al. [10] measured augmentation index in 4,001 patients of varying ages. Augmentation index is a noninvasive measurement of central pressure due to arterial stiffness and reflected pressure waves [11]. The study group found that height was inversely associated with augmentation index and posited that shorter arterial lengths resulted in earlier pressure wave reflections and greater summation of reflected pressure [10]. Evidence for this phenomenon exists in a multiethnic cross-sectional study in which taller patients were found to have lower augmentation indices and central systolic blood pressure [12]. Further epidemiological evidence supporting this phenomenon is below.

\section{Height and Blood Pressure: Literature Review}

To explore data surrounding height and blood pressure, a systematic review was undertaken independently by authors Seibert and Cochran using the search terms: "blood pressure" and "height"; excluded "adolescent," "pediatric," and "children." Additional studies cited by articles found with primary search terms, if relevant, were also included. Exclusion criteria were studies published prior to 1960 and those without English translation. In total, 6,050 abstracts resulted, of which 46 were screened and 18 sources used. Table 1 lists all studies described in order of presentation given below.

Evidence was found to support the inverse association between height and blood pressure across several large studies from diverse patient populations. Song and colleagues [13] studied 33,197 Chinese patients from the $\mathrm{Hu}$ bei Province and found that height was associated with reduced HTN in both men and women. In the INTERSALT study, cross-sectional analysis of 10,079 men and women from multiple countries by Dyer and colleagues [14] found that height was negatively associated with systolic and diastolic blood pressure. Das Gupta et al. [15] in a cross-sectional study of 13,393 Nepalese men and women found hypertension to be significantly associated with shorter height. Langenberg et al. [16] in a prospective birth cohort study of 3,035 men and women in the UK found that SBP was inversely associated with height and leg length and that DBP was not associated. In a cross- 


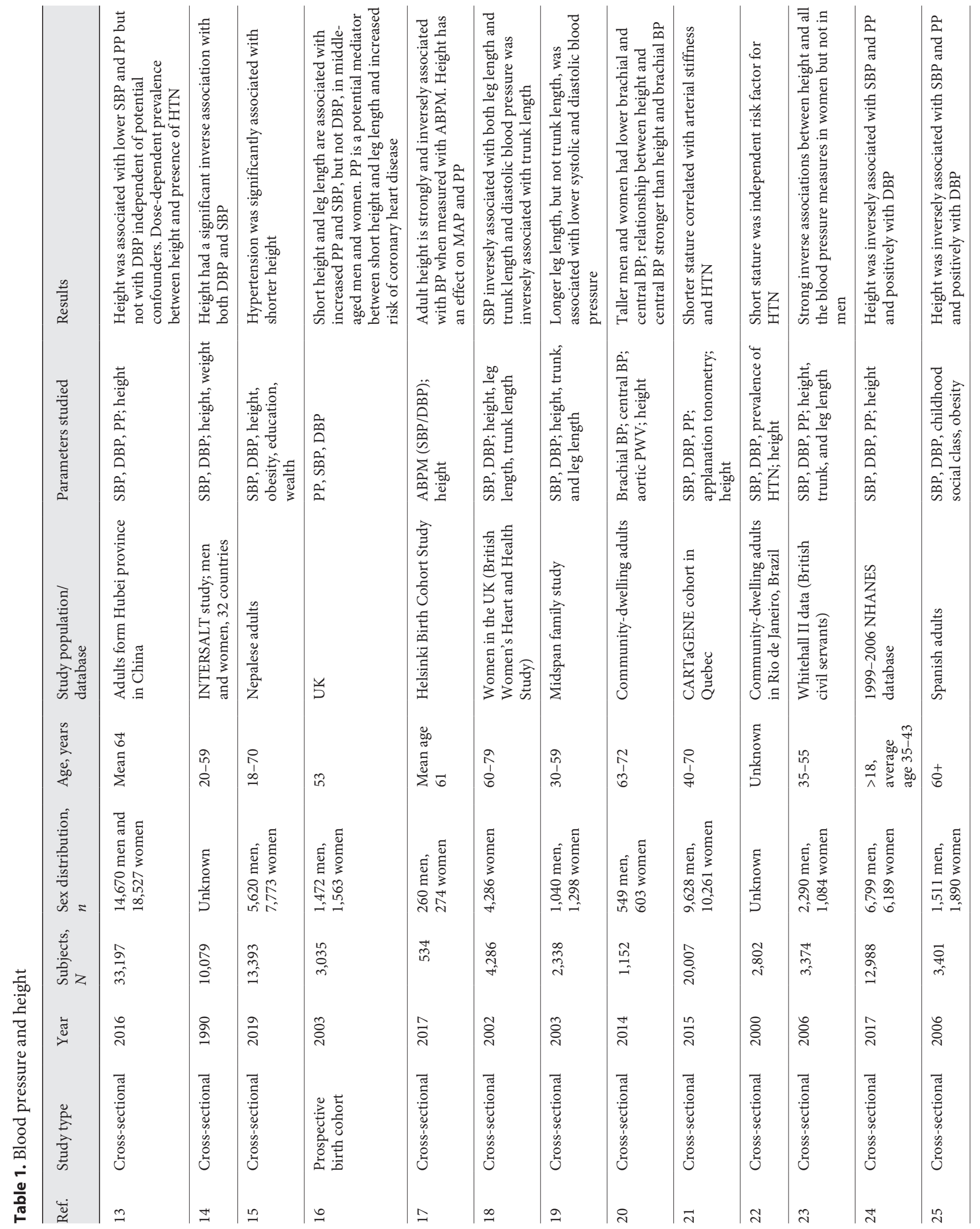




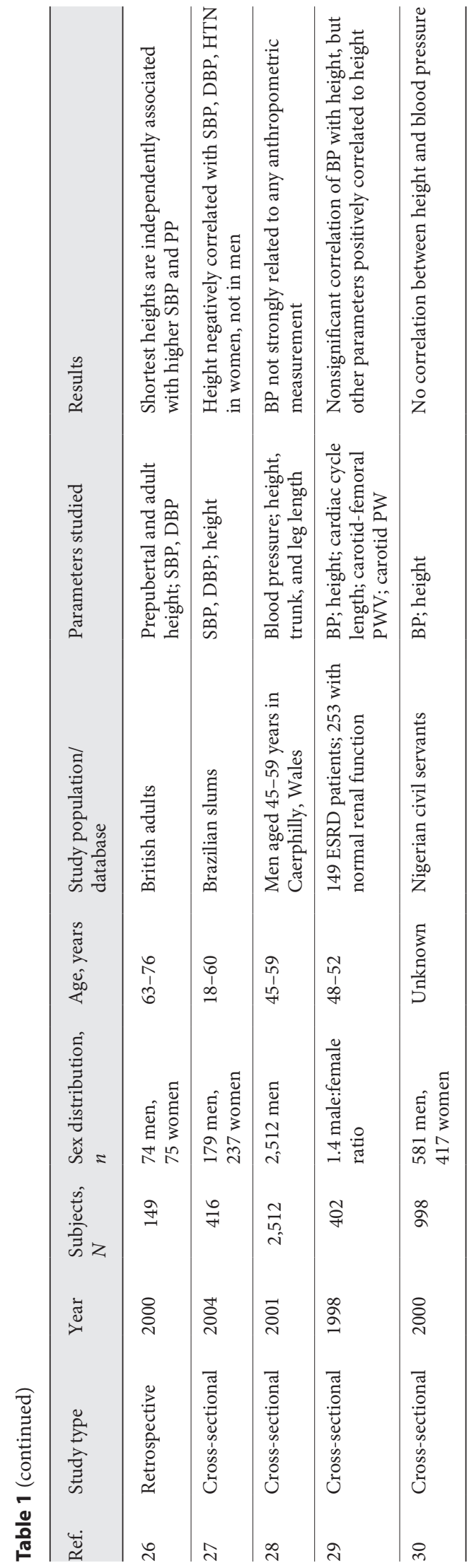

sectional study by Korhonen et al. [17] of 534 adults who were not taking any vasoactive medications, height was found to be inversely associated with ambulatory 24-h blood pressure. Some authors measured trunk length and leg length (femur) rather than height. Lawlor et al. [18] found that SBP was inversely associated with both leg length and trunk length (general surrogates of height), but only trunk length was inversely associated with DBP in a cross-sectional study of 4,286 elderly women (leg length was inversely associated with DBP but not to a statistically significant extent) [18]. In a cross-sectional study of 2,338 adults, height was inversely associated with SBP and DBP (though not to statistically significant levels). Association of leg length demonstrated the same relationship but reached statistical significance [19]. Reeve and colleagues [20] performed a detailed cross-sectional analysis of 1,152 men and women, measuring both peripheral and central blood pressure as well as additional hemodynamic measurements. They found nonsignificant inverse associations between peripheral SBP but significant inverse relationship between central systolic blood pressure as well as augmentation index. What is even more interesting is that the authors noted the percentage of the shorter cohort of patients with hypertension and on vasoactive drugs was significantly higher than the taller cohort. Had hemodynamic measurements been made without patient hemodynamics influenced by drugs, the results may have been more pronounced. Another interesting point is whether central blood pressure measurements are more sensitive and may have detected a relationship in other studies that found opposite or no significant relationship between height and blood pressure [20]. In some studies, such as the 20,007-patient Canadian cross-sectional study by ElBikai et al. [21], height was inversely associated with hypertension, especially when adjusted for use of antihypertensive medication.

Some researchers found differential relationships of blood pressure to height depending on patient sex, association of SBP and PP, but not DBP, or inverse associations that did not reach statistical significance. Sichieri et al. [22], in a cross-sectional study of 2,802 adults, found that height was inversely associated with hypertension in women but not men. Similarly, Ferrie and colleagues [23] found an inverse association between height and all BP parameters in a cross-sectional study of 3,374 adults for women but not for men. Bourgeois et al. [24] analyzed 12,988 men and women from the USA in the NHANES database and found that while SBP was inversely associated with height in older men and women, DBP was positively associated and MAPs and were not significantly different. Regidor and colleagues [25] 
found that $\mathrm{BP}$ was inversely associated with height, but not independently so, in a cross-sectional study of 4,009 men and women. Montgomery et al. [26], in a retrospective cohort study of 149 British men and women, found that height was inversely associated with blood pressure. However, the association was not statistically significant. Florencio et al. [27] found also, in a study of 416 people in Brazil, that height was negatively correlated with SBP, DBP, and HTN in women but not men.

A relatively smaller number of studies found no significant association between height and blood pressure at all. Smith and colleagues [28] did not find any statistically significant relationship between height, leg length, and trunk length versus blood pressure in a cross-sectional study of 2,512 men. Smulyan et al. [29] found no relationship between height and mean arterial blood pressure in a cross-sectional analysis of 402 men and women. No relationship between height and hypertension was found in a cross-sectional study of 2,000 Nigerian men and women by Olatunbosun and colleagues [30].

\section{Height and Response to Antihypertensive Therapies}

Overall, there are no data evaluating the relationship between height and response to blood pressure. A systematic review was conducted using search terms "height" and major antihypertensive classes. Using search terms "height" + "anti-hypertensives," 773 articles resulted but none were relevant (773 hits, 0 relevant articles). The same result was found for "height" + "beta blockers" $(252,0)$, "height" + "ace inhibitors" $(124,0)$, "height" + "angiotensin receptor blockers" $(66,0)$, "height" + "diuretics" $(266$, $0)$, and "height" + "calcium channel blockers" $(266,0)$.

\section{Height and Coronary Artery Disease}

There is a well-established relationship between height and coronary artery disease. A 2010 meta-analysis published in the European Heart Journal by Paajanen et al. [31] found an inverse relationship between height and risk of all-cause mortality, all cardiovascular disease mortality, and myocardial infarction. Subsequently, Nelson et al. [32] published an article in the NEJM in 2015 that elaborated on this topic further. In this study, genetic height (as determined by presence and quantity of certain alleles associated with height) was found to be inversely associated with coronary artery disease. The authors posit that less favorable lipid profiles associated with shorter heart

The Relationship between Adult Height and Blood Pressure explains part of this association, but that nutritional and social factors (which are also associated with adult height) make analysis difficult. These findings were corroborated by authors from the earlier (2001) Caerphilly study, in which leg length (which is determined by genetic, epigenetic, nutritional, and social exposures) was inversely associated with risk of coronary artery disease in a cohort of middle-aged Welsh men [28].

\section{Conclusions}

Significant data exist linking increased height and leg length with lower blood pressure. However, many of these studies suffer from heterogeneity in data collection, discrepant measurement techniques, and lack of control for hypertensive/vasoactive medications and have versatile patient populations. Increased stature has also been linked with reduced cardiovascular disease risk. Several plausible physiological explanations may explain these phenomena. These findings identify shorter individuals as having higher risk with regard to hypertension and cardiovascular disease but also raise novel questions - namely, whether aggressive blood pressure control mitigates the cardiovascular risk conferred by shorter height, whether taller patients are at greater risk for orthostatic side effects with aggressive treatment, and whether a variation in the antihypertensive medication response exists with respect to height. All of these questions may prove useful topics for future research that may impact approaches to hypertension treatment at the patient and population level.

\section{Statement of Ethics}

This research was conducted ethically in accordance with the World Medical Association Declaration of Helsinki.

\section{Conflict of Interest Statement}

The authors have no conflicts of interest to declare.

\section{Funding Sources}

The authors did not receive any funding.

\section{Author Contributions}

All authors had access to this manuscript and data and played a role in writing this manuscript. 


\section{References}

1 Chockalingam A, Campbell NR, Fodor JG. Worldwide epidemic of hypertension. Can J Cardiol. 2006;22(7):553-5.

2 Merai R, Siegel C, Rakotz M, Basch P, Wright J, Wong B. CDC grand rounds: a public health approach to detect and control hypertension. MMWR Morb Mortal Wkly Rep. 2016 Nov 18;65(45):1261-4.

3 The SPRINT Research Group, Wright JTJr, Williamson JD, Whelton PK, Snyder JK, Sink $\mathrm{KM}$, et al. A randomized trial of intensive versus standard blood-pressure control. N Engl J Med. 2015;372:1608-18

4 Aronow WS. Association of obesity with hypertension. Ann Transl Med. 2017;5(17):350.

5 Arnett DK, Blumenthal RS, Albert MA, Buroker AB, Goldberger ZD, Hahn EJ, et al. 2019 ACC/AHA guideline on the primary prevention of cardiovascular disease: executive summary: a report of the American College of Cardiology/American Heart Association Task Force on Clinical Practice Guidelines. Circulation. 2019 Sep 10;140(11):e596-e646.

6 Whelton PK, Carey RM, Aronow WS, Casey DEJr, Collins KJ, Dennison Himmelfarb C, et al. 2017 ACC/AHA/AAPA/ABC/ACPM/ AGS/APhA/ASH/ASPC/NMA/PCNA guideline for the prevention, detection, evaluation, and management of high blood pressure in adults: a report of the American College of Cardiology/American Heart Association Task Force on Clinical Practice Guidelines. Hypertension. 2018;71(6):1269-324

7 Williams B, Mancia G, Spiering W, Agabiti Rosei E, Azizi M, Burnier M, et al. 2018 ESC/ ESH Guidelines for the management of arterial hypertension. Eur Heart J. 2018;39(33): 3021-104.

8 Victor RG. Systemic hypertension: mechanisms and diagnosis. In: Braunwald's heart disease: a textbook of cardiovascular medicine. 11th ed. Philadelphia, PA: Elsevier; 2019. p. 910-27.

9 Franklin SS. Hypertension in older people: part 1. J Clin Hypertens. 2006;8(6):444-9.

10 McEniery CM, Yasmin, Hall IR, Qasem A Wilkinson IB, Cockcroft JR. Normal vascular aging: differential effects on wave reflection and aortic pulse wave velocity: the AngloCardiff Collaborative Trial (ACCT). J Am Coll Cardiol. 2005;46(9):1753-60.

11 Wilkinson IB, MacCallum H, Flint L, Cockcroft JR, Newby DE, Webb DJ. The influence of heart rate on augmentation index and central arterial pressure in humans. J Physiol. 2000;525 Pt 1(Pt 1):263-70.
12 Eeftinck Schattenkerk DW, van Gorp J, Snijder MB, Zwinderman AH, Agyemang CO, Peters RJ, et al. Correction: ethnic differences in arterial wave reflection are mostly explained by differences in body height-crosssectional analysis of the HELIUS study. PLoS ONE. 2016;11(12): 0168620.

13 Song L, Shen L, Li H, Liu B, Zheng X, Liang Y, et al. Height and prevalence of hypertension in a middle-aged and older Chinese population. Sci Rep. 2016;6:39480.

14 Dyer AR, Elliott P, Shipley M. Body mass index versus height and weight in relation to blood pressure. Findings for the 10,079 persons in the INTERSALT Study. Am J Epidemiol. 1990;131(4):589-96.

15 Das Gupta R, Haider SS, Hashan MR, Rahman MA, Sarker M. Association between height and hypertension in the adult Nepalese population: findings from a nationally representative survey. Health Sci Rep. 2019;2(12): e141.

16 Langenberg C, Hardy R, Kuh D, Wadsworth ME. Influence of height, leg and trunk length on pulse pressure, systolic and diastolic blood pressure. J Hypertens. 2003;21(3):537-43.

17 Korhonen PE, Kautiainen H, Eriksson JG. The shorter the person, the higher the blood pressure: a birth cohort study. J Hypertens. 2017;35(6):1170-7.

18 Lawlor DA, Ebrahim S, Davey Smith G. The association between components of adult height and type II diabetes and insulin resistance: British women's heart and health study. Diabetologia. 2002;45(8):1097-106.

19 Gunnell D, Whitley E, Upton MN, McConnachie A, Smith GD, Watt GC. Associations of height, leg length, and lung function with cardiovascular risk factors in the midspan family study. J Epidemiol Community Health. 2003;57(2):141-6.

20 Reeve JC, Abhayaratna WP, Davies JE, Shar man JE. Central hemodynamics could explain the inverse association between height and cardiovascular mortality. Am J Hypertens. 2014;27(3):392-400

21 El-Bikai R, Tahir MR, Tremblay J, Joffres M, Šeda O, Šedová L, et al. Association of agedependent height and bone mineral density decline with increased arterial stiffness and rate of fractures in hypertensive individuals. J Hypertens. 2015;33(4):727-35.
22 Sichieri R, Siqueira KS, Pereira RA, Ascherio A. Short stature and hypertension in the city of Rio de Janeiro, Brazil. Public Health Nutr. 2000;3(1):77-82.

23 Ferrie JE, Langenberg C, Shipley MJ, Marmot MG. Birth weight, components of height and coronary heart disease: evidence from the Whitehall II study. Int J Epidemiol. 2006; 35(6): 1532-42.

24 Bourgeois B, Watts K, Thomas DM, Carmichael $\mathrm{O}, \mathrm{Hu} \mathrm{FB}$, Heo M, et al. Associations between height and blood pressure in the United States population. Medicine. 2017; 96(50):e9233.

25 Regidor E, Banegas JR, Gutiérrez-Fisac JL, Domínguez V, Rodríguez-Artalejo F. Influence of childhood socioeconomic circumstances, height, and obesity on pulse pressure and systolic and diastolic blood pressure in older people. J Hum Hypertens. 2006;20(1): 73-82.

26 Montgomery SM, Berney LR, Blane D. Prepubertal stature and blood pressure in early old age. Arch Dis Child. 2000;82(5):358-63.

27 Florencio TT, Ferreira HS, Cavalcante JC, Sawaya AL. Short stature, obesity and arterial hypertension in a very low income population in North-Eastern Brazil. Nutr Metab Cardiovasc Dis. 2004;14(1):26-33.

28 Smith GD, Greenwood R, Gunnell D, Sweetnam P, Yarnell J, Elwood P. Leg length, insulin resistance, and coronary heart disease risk: the Caerphilly study. J Epidemiol Community Health. 2001;55(12):867-72.

29 Smulyan H, Marchais SJ, Pannier B, Guerin AP, Safar ME, London GM. Influence of body height on pulsatile arterial hemodynamic data. J Am Coll Cardiol. 1998;31(5):1103-9.

30 Olatunbosun ST, Bella AF. Relationship between height, glucose intolerance, and hypertension in an urban African black adult population: a case for the "thrifty phenotype" hypothesis? J Natl Med Assoc. 2000;92(6): 265-8.

31 Paajanen TA, Oksala NK, Kuukasjärvi P, Karhunen PJ. Short stature is associated with coronary heart disease: a systematic review of the literature and a meta-analysis. Eur Heart J. 2010;31(14):1802-9.

32 Nelson CP, Hamby SE, Saleheen D, Hopewell JC, Zeng L, Assimes TL, et al. Genetically determined height and coronary artery disease. N Engl J Med. 2015;372(17):1608-18. 